\title{
An Algorithm for the Orientation of Complete Bipartite Graphs
}

\author{
Lingqi Zhao ${ }^{1}$, Mujiangshan Wang ${ }^{2}$, Xuefei Zhang ${ }^{3}$, Yuqing Lin², Shiying Wang ${ }^{4, *}$ \\ ${ }^{1}$ Institute of Discrete Mathematics, College of Computer Science and Technology, Inner Mongolia University for Nationalities, \\ Tongliao 028043 PR China \\ ${ }^{2}$ School of Electrical Engineering and Computer Science, The University of Newcastle NSW 2308, Australia \\ ${ }^{3}$ School of Mathematical Sciences, Shanxi University, Taiyuan, Shanxi 030006, PR China \\ ${ }^{4}$ School of Mathematics and Information Science, Henan Normal University, Xinxiang, Henan 453007, PR China \\ ${ }^{*}$ Corresponding author
}

Abstract-Let $G$ be a graph with vertex set $V(G)$ and edge set $E(G)$. We consider the problem of orienting the edges of a complete bipartite graph $K_{n, n}$ so only two different in-degrees $a$ and $b$ occur. An obvious necessary condition for orienting the edges of $G$ so that only two in-degrees $a$ and $b$ occur, is that there exist positive integers $s$ and $t$ satisfying $s+t=|V(G)|$ and $a s+b t=|E(G)|$. In this paper, we show that the necessary condition is also sufficient for a complete bipartite graph $K_{n, n}$. Furthermore, we give the algorithms of orientations with only two in-degrees of $K_{n, n}$.

Keywords-complete bipartite graph; orientation; algorithm

\section{INTRODUCTION}

An orientation $D=(V(D), A(D))$ of an undirected graph $G=(V(G), E(G))$ is a digraph obtained by replacing each undirected edge $e \in E$ with an arc from one end vertex of $e$ to the other. In the oriented problem, we are asked whether $G$ has an orientation satisfying some conditions. This is a basic problem in combinatorial optimization, and many beautiful results have been produced so far. Chen et al. [3] studied orientations of graphs satisfying the Ore condition. Fukunaga [4] investigated graph orientations with set connectivity requirements. Miao and Lin [7] gave strong orientations of complete $k$-partite graphs achieving the strong diameter. The main purpose of this paper is to orient $K_{n, n}$ with $a$ or $b$ arrowheads directed towards each vertex.

Usually a digraph has many different in-degrees. This paper is to orient graph $K_{n, n}$ achieving only two in-degrees. This kind of oriented problem is useful in practice. Buhler et al. [1] considered the problem of orienting the edges of the $n$ dimensional hypercube so that only two in-degrees occur for finding strategies for specific hat guessing games.

Let $D$ be a digraph, for any $u v \in A(D)$, we say that $u$ dominates $v$ (or $v$ is dominated by $u$ ) and denote it by $u \rightarrow v$.
For any $v \in V(D)$, the in-degree of $v$ is denoted by $d^{-}(v)=|\{u \in V(D): u v \in A(D)\}|$ and the out-degree of $v$ is denoted by $d^{+}(v)=|\{u \in V(D): v u \in A(D)\}|$. For disjoint subsets $X$ and $Y$ of $V(D), X \rightarrow Y$ means that every vertex of $X$ dominates every vertex of $Y$, and we define $[X, Y]=\{x y \in A(D): x \in X, y \in Y\} \quad$. For graph-theoretical terminology and notation not defined here we follow[2, 6].

In this paper, $K_{n, n}$ is oriented to a digraph so that only two in-degrees $a$ and $b$ occur. For convenience, let $[a, b]_{n}$ be a shorthand for the problem of realizing an orientation of $K_{n, n}$ whose only in-degrees are $a$ or $b$. In Section 2, we give a necessary and sufficient condition such that $[a, b]_{n}$ is realizable. In Section 3, we give some specified algorithms to construct the required orientations of $K_{n, n}$.

\section{MAIN RESULTS}

Lemma 2.1. Given a positive integer $n$, let $K_{n, n}$ be a complete bipartite graph. For $a, b \in\{0,1,2, \ldots, n\}$, if $[a, b]_{n}$ is realizable, then there exist positive integers $s$ and $t$ satisfying the following two equations:

$$
\left\{\begin{array}{c}
s+t=2 n, \\
a s+b t=n^{2}
\end{array}\right.
$$

Proof. Let $[a, b]_{n}$ be realizable. Then there exixts an oriented graph whose only in-degrees are $a$ or $b$. Let the indegree of $s$ vertices in $K_{n, n}$ be $a$. Then the in-degree of the remaining $(2 n-s)$ vertices is $b$. Therefore, $s+t=\left|V\left(K_{n, n}\right)\right|=2 n$, and $a s+b t=\left|E\left(K_{n, n}\right)\right|=n^{2}$, where $t=n-s$.

Let $G$ be a nonoriented graph. For $U \subseteq V(G)$, denote the number of edges which have their both end-vertices in $U$ by $e(U)$. 
Lemma 2.2. [5] Given a nonoriented graph $G$ whose vertices are labeled $v_{1}, v_{2}, \cdots, v_{n}$ and to whose vertices are associated non-negative integers $v\left(v_{1}\right), v\left(v_{2}\right), \cdots, v\left(v_{n}\right)$, respectively; then, $G$ is orientable with $v\left(v_{i}\right)$ arrowheads directed toward vertex $v_{i}$ (for $i \in\{1,2, \ldots, n\}$ ) if and only if $\sum_{v \in V(G)} v(v)=|E(G)|$ and

$$
e(U) \leq \sum_{v \in U} v(v) \text { for each } U \subseteq V(G)
$$

Lemma 2.3. Given three positive integers $n, s$ and $t$, let $K_{n, n}$ be a complete bipartite graph and let $a, b \in\{0,1,2, \ldots, n\}$ with $a \leq b$. If $a, b, s, t$ satisfy the following two equations:

$$
\left\{\begin{array}{l}
s+t=2 n, \\
a s+b t=n^{2}
\end{array}\right.
$$

Then $[a, b]_{n}$ is realizable.

Proof. Case 1. $s>t$.

By $a \leq b$ and $a, b \in\{0,1,2, \ldots, n\}, n-a \geq n-b$ and $n-a, n-b \in\{0,1,2, \ldots, n\}$. We can deduce that $(n-a) s+(n-b) t=(n-a) s+(2 n-s)(n-b)=2 n^{2}-[a s+b(2 n-s)]$ $=2 n^{2}-n^{2}=n^{2}$. Set $a^{\prime}=n-b, b^{\prime}=n-a, s^{\prime}=t, t^{\prime}=s$. Now, $a^{\prime} \leq b^{\prime}$ and $s^{\prime}<t^{\prime}$. Then $\$ a^{\prime}, b^{\prime}, s^{\prime}, t^{\prime} \$$ satisfy the conditions of Case 1. By the proof of Case $1,\left[a^{\prime}, b^{\prime}\right]_{n}$ is realizable. Then $K_{n, n}$ has an orientation $D$ whose only indegrees are $a^{\prime}$ or $b^{\prime}$. We consider the digraph $D^{\prime}$ obtained by reversing all the arcs in $D$. Note that $K_{n, n}$ is $n$-regular. Then $\left[n-a^{\prime}, n-b^{\prime}\right]_{n}$ is realizable, i.e. $[b, a]_{n}$ is realizable. So $[a, b]_{n}$ is realizable.

Case 2. $s \leq t$.

The proof of this case is similar to Case 1.

Lemma 2.4. Given three positive integers $n, s$ and $t$, let $K_{n, n}$ be a complete bipartite graph and let $a, b \in\{0,1,2, \ldots, n\}$ with $a>b$. If $a, b, s, t$ satisfy the following two equations:

$$
\left\{\begin{array}{c}
s+t=2 n, \\
a s+b t=n^{2}
\end{array}\right.
$$

Then $[a, b]_{n}$ is realizable.

Proof. By $a>b$ and $a, b \in\{0,1,2, \ldots, n\}, n-a<n-b$ and $n-a, n-b \in\{0,1,2, \ldots, n\}$. We can deduce that $(n-a) s+(n-b) t=(n-a) s+(2 n-s)(n-b)=2 n^{2}-[a s+b(2 n-s)]$
$=2 n^{2}-n^{2}=n^{2}$. Then $n-a$ and $n-b$ satisfy the conditions of Lemma 2.3. By Lemma 2.3, $[n-a, n-b]_{n}$ is realizable. Then $K_{n, n}$ has an orientation $D$ whose only in-degrees are $n-a$ or $n-b$. We consider the digraph $D^{\prime}$ obtained by reversing all the arcs in $D$. Note that $K_{n, n}$ is $n$-regular. Then $[n-(n-a), n-(n-b)]_{n}=[a, b]_{n}$ is realizable.

By Lemmas 2.1, 2.3 and 2.4, we can obtain the following theorem directly.

Theorem 2.5. Given a positive integer $n$, let $K_{n, n}$ be a complete bipartite graph. For $a, b \in\{0,1,2, \ldots, n\},[a, b]_{n}$ is realizable if and only if there exist positive integers $s$ and $t$ satisfying the following two equations:

$$
\left\{\begin{array}{c}
s+t=2 n, \\
a s+b t=n^{2} .
\end{array}\right.
$$

Corollary 2.6. Given a positive integer $n$, let $K_{n, n}$ be a complete bipartite graph. For $a, b \in\{0,1,2, \ldots, n\}$, the following results hold:

(a) if $[a, b]_{n}$ is realizable, then $[n-a, n-b]_{n}$ is realizable;

(b) $[0, n]_{n}$ is realizable;

(c) if $n$ is even, then $\left[\frac{n}{2}, \frac{n}{2}\right]_{n}$ is realizable.

\section{ORIENTATION ALgORITHMS OF $K_{n, n}$}

In Section 2, we have proved that $K_{n, n}$ admits the orientation with only two in-degrees. In this section, we will show how to orient $K_{n, n}$ by specified algorithms. By the proofs of Lemmas 2.3 and 2.4, it is enough to consider the case where $a \leq b$ and $s \leq t$.

Specially, $K_{1,1}$ has an orientation $D$ whose only in-degrees are 0 or 1 . Then $[0,1]_{1}$ is obviously realizable. In the following, suppose that $n \geq 2$.

If $a=b$, then, by the equations (2) and (3), $a=b=\frac{n}{2}$. Note that $a$ is an integer and $a=\frac{n}{2}$. Therefore, $n$ is even. Combining this with the fact that the degree of each vertex in $K_{n, n}$ is $n, K_{n, n}$ admits an Euler tour. By the definition of Euler tour, there exists an oriented graph of $K_{n, n}$ whose only in-degree is $\frac{n}{2}$. Then $\left[\frac{n}{2}, \frac{n}{2}\right]_{n}$ is realizable. Next assume that $a<b$. By the equations (2) and (3), we have $s=\frac{n(2 b-n)}{b-a}$ 
and $t=\frac{n(n-2 a)}{b-a}$. Combining this with the fact that $s$ and $t$ are positive integers, $a<\frac{n}{2}<b$.

By $b>\frac{n}{2}, b>n-b$. Since $s \leq t$ and $s+t=2 n, n-s \geq 0$. We can deduce that $n^{2}=a s+b t=a s+b(2 n-s)=a s+b n+b(n-s) \geq$ $a s+b n+(n-b)(n-s)=a s+b n+n^{2}-b n-s n+b s=(a+b-n) s+n^{2}$ Hence $(a+b-n) s \leq 0$. Combining this with $s>0, a+b \leq n$.

Case 1. $a+b=n$.

Let $(X, Y)$ be a bipartition of $K_{n, n}$ with $X=\left\{u_{0}, u_{1}, \ldots, u_{n-1}\right\}$ and $Y=\left\{v_{0}, v_{1}, \ldots, v_{n-1}\right\}$. By $a+b=n$, $s=\frac{n(2 b-n)}{b-a}=\frac{n(2 b-n)}{b-(n-b)}=\frac{n(2 b-n)}{2 b-n}=n \quad, \quad$ i.e., $\quad s=n$. Combining this with $s+t=2 n, t=n$. Then $s=t=n$. Conversely, if $s=t$, then By the equations (2) and (3), we have $s=t$ and $a+b=n$. If $a=0$, then $b=n$. Construct a special orientation such that $X \rightarrow Y$. Then the in-degree of each vertex in $X$ is 0 and the in-degree of each vertex in $Y$ is $n$. Therefore, $[0, n]_{n}$ is realizable. Next, assume that $a>0$. For any $u_{i} \in X \quad$, orient $\quad v_{(i+l)(\bmod n)} \rightarrow u_{i}$ for each $l=0,1, \ldots, a-1$. We orient the remaining edges which are incident to $u_{i}$ towards $Y$. Now, we obtain an oriented graph $D$. The in-degree of each vertex of $X$ in $D$ is $a$. For any $v_{i} \in Y$, by the definition of $D, \quad v_{i} \rightarrow u_{(i-l)(\bmod n)}$ for each $l=0,1, \ldots, a-1$. The out-degree of each vertex of $Y$ in $D$ is $a$, and the in-degree of each vertex of $Y$ in $D$ is $n-a$. Only two in-degrees $a$ and $n-a$ occur in $D$. Set $b=n-a$. Therefore, $[a, b]_{n}$ is realizable. Then we can obtain the following proposition directly.

Proposition 3.1. Let $a<b$ and $a+b=n$. Then the oriented graph which is obtained by the above method has only two in-degrees $a$ and $b$.

Case 2. $a+b<n$.

In this case, $s<t$. By $a+b<n$ and $a<b$, $n-s=n-\frac{n(2 b-n)}{b-a}=\frac{(b-a) n-n(2 b-n)}{b-a}=\frac{n(n-a-b)}{b-a}>0$, i.e., $s<n$. Combining this with $s+t=2 n, t>n$. Then $s<n<t$.

First, assume that $a=0$. By $a+b<n, b<n$. If $s \geq b$, $n^{2}=a s+b t=a s+b(2 n-s)=a s+b n+b(n-s)<a s+s n+n(n-s)=n^{2}$. This is a contradiction. So $s<b$.

Let $(X, Y)$ be a bipartition of $K_{n, n}$ with $X=\left\{u_{0}, u_{1}, \ldots, u_{n-1}\right\}, \quad Y=Y_{1} \cup Y_{2}, Y_{1} \cap Y_{2}=\varnothing$, and let $Y_{1}=\left\{v_{0}, v_{1}, \ldots, v_{b-1}\right\} \quad, \quad Y_{2}=\left\{z_{0}, z_{1}, \ldots, z_{n-b-1}\right\} \quad$. Orient
$Y_{1} \rightarrow X \rightarrow Y_{2}$. Now, we obtain an oriented graph $D$ (see Fig.1). The in-degree of each vertex $u_{i}$ of $X$ in $D$ is $b$. The in-degree of each vertex $v_{i}$ of $Y_{1}$ in $D$ is 0 and the in-degree of each vertex $z_{j}$ of $Y_{2}$ in $D$ is $n$. Denote the vertex set $\left\{v_{0}, \ldots, v_{b-s-1}\right\} \subseteq Y_{1}$ by $Y_{1}^{\prime}$. By $s<b,\left|Y_{1}^{\prime}\right| \geq 1$.

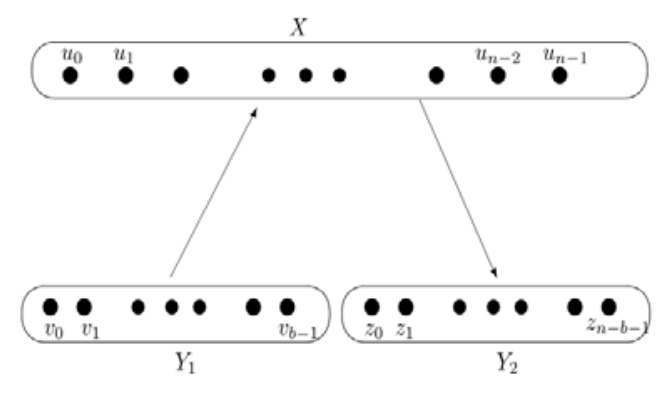

FIGURE I. THE ORIENTED GRAPH D

\section{Algorithm 3.1.}

INPUT: the above oriented graph $D$ with three in-degrees $0, b$ and $n$.

0 . Set $l_{x}:=0$ for every $x=0,1, \ldots, b-s-1, r_{y}:=n$ for every $y=0,1, \ldots, n-b-1, i:=0, j:=0$.

1. If $l_{i} \geq b, i:=i+1$.

2. If $r_{j} \leq b, j:=j+1$.

3. Choose $u \in X$ satisfying $v_{i} \rightarrow u \rightarrow z_{j}$ in $D$. Reverse $v_{i} \rightarrow u \rightarrow z_{j}$ in $D$. Obtain $D^{*} . D:=D^{*}$.

4. Set $l_{i}:=l_{i}+1$ and $r_{j}:=r_{j}-1$.

5. If $i=b-s-1$ and $l_{i}=b$, output $D$. Otherwise, go to step 1.

Theorem 3.2. Let $a=0$ and $a+b<n$. Then Algorithm 3.1 outputs $D$ which has only two in-degrees 0 and $b$.

\section{Algorithm 3.2}

INPUT: the above oriented graph $D$ with four in-degrees $a, b, n-a$ and $n-b$.

0 . Set $l_{x}:=n-a$ for every $x=0,1, \ldots, s-1, r_{y}:=n-b$ for every $y=0,1, \ldots, n-s-1, i:=0, j:=0$.

1. If $r_{j} \geq b, j:=j+1$.

2. If $l_{i} \leq b, i:=i+1$.

3. $w \in\left\{w_{j}, w_{(j-1)(\bmod (n-s))}, w_{(j-2)(\bmod (n-s))}, \ldots, w_{(j-b+1)(\bmod (n-s))}\right\}$ satisfying $z_{j} \rightarrow w \rightarrow v_{i}$ in $D$. Reverse $z_{j} \rightarrow w \rightarrow v_{i}$ in $D$. Obtain $D^{*}$. $D:=D^{*}$. 
4. Set $r_{j}:=r_{j}+1$ and $l_{i}:=l_{i}-1$.

5. If $j=n-s-1$ and $r_{j}=b$, output $D$. Otherwise, go to step 1.

Theorem 3.3. Let $0<a<b \leq n-s, \quad a \leq s<t$ and $a+b<n$. Then Algorithm 3.2 outputs $D$ which has only two in-degrees $a$ and $b$.

\section{Algorithm 3.3}

INPUT: the above oriented graph $D$ with four in-degrees $a, b, n+s-b$ and $s-a$.

0 . Set $l_{x}:=s-a$ for every $x=0,1, \ldots, s-1, r_{y}:=n+s-b$ for every $y=0,1, \ldots, n-s-1, i:=0, j:=0$.

1. If $l_{i} \geq b, i:=i+1$.

2. If $r_{j} \leq b, j:=j+1$.

3. Choose $q \in\left\{u_{i}, u_{(i-1)(\bmod s)}, \ldots, u_{(i-a+1)(\bmod s)}\right\} \cup$ $\left(X_{2} \backslash\left\{w_{j}, w_{(j-1)(\bmod (n-s))}, \ldots, w_{(j-b+s+1)(\bmod (n-s))}\right\}\right) \quad$ satisfying $v_{i} \rightarrow q \rightarrow z_{j}$ in $D$. Reverse $v_{i} \rightarrow q \rightarrow z_{j}$ in $D$. Obtain $D^{*}$. $D:=D^{*}$.

4. Set $l_{i}:=l_{i}+1$ and $r_{j}:=r_{j}-1$.

5. If $i=s-1$ and $l_{i}=b$, output $D$. Otherwise, go to step 1 .

Theorem 3.4. Let $0<a<b, n-s<b, s<b, a \leq s<t$ and $a+b<n$. Then Algorithm 3.3 outputs D which has onlu two in-degrees $a$ and $b$.

\section{Algorithm 3.4.}

INPUT: the above oriented graph $D$ with five in-degrees $a, b, n-s, s$, and $n$.

0 . Set $l_{x}:=n-s$ for every $x=0,1, \ldots, a-1, r_{y}:=s$ for every $\quad y=0,1, \ldots, b-1 \quad, \quad r_{k}:=n \quad$ for every $k=b, b+1, \ldots, n-a-1, i:=0, j:=0$.

1. If $r_{j} \leq b, j:=\min \left\{\beta: r_{\beta}>b, j+1 \leq \beta \leq n-a-1\right\}$.

2. If $l_{i} \geq b, i:=i+1$.

3. Choose $u \in X_{1}$ satisfying $v_{i} \rightarrow u \rightarrow z_{j}$ in $D$. Reverse $v_{i} \rightarrow u \rightarrow z_{j}$ in $D$. Obtain $D^{*} . D:=D^{*}$.

4. Set $r_{j}:=r_{j}-1$ and $l_{i}:=l_{i}+1$.

5. If $j=n-a-1$ and $r_{j}=b$, output $D$. Otherwise, go to step 1.

Theorem 3.5. Let $0<a<b, n-s<b, s \geq b, a \leq s<t$ and $a+b<n$. Then Algorithm 3.4 outputs $D$ which has only two in-degrees $a$ and $b$.

\section{Algorithm 3.5.}

INPUT: the above oriented graph $D$ with five in-degrees $a, b, n-a, n+s-b-a$, and $n-b$.

0 . Set $l_{x}:=n-a$ for every $x=0,1, \ldots, a-1$, $r_{y}:=n+s-b-a$ for every $y=0,1, \ldots, b-a-1, r_{k}:=n-b$ for every $k=b-a, b-a+1, \ldots, n-a-1, i:=0, j:=0$.

1. If $r_{j} \geq b, j:=j+1$.

2. If $l_{i} \leq b, i:=i+1$.

3.

$w \in\left\{w_{j}, w_{(j-1)(\bmod (n-a))}, w_{(j-2)(\bmod (n-a))}, \ldots, w_{(j-b+1)(\bmod (n-a))}\right\}$

satisfying $z_{j} \rightarrow w \rightarrow v_{i}$ in $D$. Reverse $z_{j} \rightarrow w \rightarrow v_{i}$ in $D$. Obtain $D^{*}$. $D:=D^{*}$.

4. Set $r_{j}:=r_{j}+1$ and $l_{i}:=l_{i}-1$.

5. If $j=n-a-1$ and $r_{j}=b$, output $D$. Otherwise, go to step 1.

Theorem 3.6. Let $0<a<b, \quad s<a, s<t$ and $a+b<n$. Then Algorithm 3.5 outputs $D$ which has only two in-degrees $a$ and $b$.

\section{ACKNOWLEDGEMENTS}

This work is supported by the National Science Foundation of China $(61370001,61261025,61262018)$ and the Natural Science Foundation of Inner Mongolia (2014MS0116, 2014MS0110).

\section{REFERENCES}

[1] Joe Buhler, Steve Butler, Ron Graham, Eric Tressler: Hypercube orientations with only two in-degrees. Journal of Combinatorial Theory, Series A 118 (6), 1695-1702 (2011)

[2] J.A. Bondy, U.S.R. Murty: Graph Theory. Springer, New York (2007)

[3] Meirun Chen, Xiaofeng Guo, Hao Li: Lower and upper orientable strong diametera of graphs satisfying the ore condition. Applied Mathematices Letters 22 (7), 994-997 (2009)

[4] Takuro Fukunaga: Graph orientations with set connectivity requirements. Discrete Mathematics 312 (15), 2349-2355 (2012)

[5] S.L. Hakimi: On the degrees of the vertices of a directed graph. Journal of the Franklin Institute 279 (4), 290-308 (1965)

[6] J.B. Jensen, G. Gutin: Digraphs Theory. Algorithms and Applications, Springer, New York (2007)

[7] Huifang Miao, Guoping Lin: Strong orientations of complete \$k\$-partite graphs achieving the strong diameter. Information Processing Letters 110 (6), 206-210 (2010) 